\title{
Tumor burden in Hodgkin's lymphoma can be reliably estimated from a few staging parameters
}

\author{
PAOLO G. GOBBI ${ }^{1}$, MANUELA BERGONZI ${ }^{1}$, EMILIO BASSI ${ }^{2}$, FRANCESCO MERLI ${ }^{3}$, \\ CHIARA CORIANI $^{4}$, CATERINA STELITANO ${ }^{5}$, EMILIO IANNITTO ${ }^{6}$ and MASSIMO FEDERICO ${ }^{7}$ \\ ${ }^{1}$ Department of Internal Medicine and Gastroenterology, ${ }^{2}$ Institute of Radiology, University of Pavia, \\ IRCCS S. Matteo Policlinico Foundation, 27100 Pavia; Departments of ${ }^{3}$ Hematology and ${ }^{4}$ Radiology, Arcispedale \\ S. Maria Nuova, 42100 Reggio Emilia; ${ }^{5}$ Department of Hematology, Riuniti Hospital, 89100 Reggio Calabria; \\ ${ }^{6}$ Department of Hematology, SS. Annunziata Hospital, 74100 Taranto; ${ }^{7}$ Department of Oncology, \\ University of Modena and Reggio Emilia, Policlinico of Modena, 4110 Modena, Italy
}

Received February 17, 2012; Accepted April 12, 2012

DOI: $10.3892 /$ or.2012.1892

\begin{abstract}
The relative tumor burden (rTB), the tumor burden normalized to body surface area, is of prime clinical and prognostic value in Hodgkin's lymphoma. However, its measurement is rather complicated and a bedside computation cannot be proposed. We investigated the possibility of estimating, instead of measuring, rTB from elementary parameters of the initial staging. The rTB of 507 patients, treated with therapeutic protocols of the Gruppo Italiano Studio Linfomi according to their staging characteristics, was measured through their pre-therapy computed tomographies. The relationships between $\mathrm{rTB}$ and staging characteristics were analyzed with simple and multiple regressions both in a training sample (254 patients) for a selection of predictive parameters, and in a test sample (253 patients) for validation of the results. The number of involved sites, bulky mass and the IPI score were the variables best related to rTB. The resulting final equation estimated $\mathrm{rTB}=-4.3+8.3 \times \mathrm{IPI}^{2}+22.7 \times$ [no. of involved sites ( +3 if a bulky mass is present)]\} provided the maximal approximation to the measured $\mathrm{rTB}\left(\mathrm{R}^{2}=0.671\right)$. The validity of the equation was confirmed on the test sample and the predictive superiority of the estimated rTB over IPI was still evident in terms of failure-free survival in both groups of patients. The estimated rTB is accurate enough to retain most of the prognostic advantage of the measured rTB over the IPI score. It can be easily calculated, allows a valid approximation of the measured rTB, and can be proposed as a useful tool for clinical research and practice.
\end{abstract}

Correspondence to: Professor Paolo G. Gobbi, Clinica Medica I, University of Pavia, IRCCS S. Matteo Policlinico, P.le Golgi 2, 27100 Pavia, Italy

E-mail: gobbipg@smatteo.pv.it

Key words: Hodgkin's lymphoma, tumor burden, clinical staging, prognostic factors, failure-free survival

\section{Introduction}

Many of the clinical parameters used for the initial staging of patients with Hodgkin's lymphoma have a well-known, partly independent prognostic value and are variably related to the tumor burden (TB). This is the case of clinical stage, bulky mass, number of involved regions, inguinal lymphadenopathy, number of splenic nodules, and numerous serologic indicators, including lactate dehydrogenase, $\beta 2$-microglobulin, serum albumin, hemoglobin, white blood cell count, peripheral lymphocyte count, erythrosedimentation rate, and soluble CD30 and CD54 concentration (1,2). All these factors can be individually considered as vague and inaccurate surrogate indicators for TB. However, in Hodgkin's lymphoma, as in several other tumors, a direct measure of TB, even if only estimated, is invariably prognostically superior to any other indicator. In some studies conducted between 1986 and 1992 Specht et al $(3,4)$ devised and extensively applied an indirect technique for estimating the TB. The method was semiquantitative, rather complex, with some step of subjectivity, and relied partly on the evaluation of abdominal lymphangiography, a radiologic examination that has now been completely abandoned. Despite the potential inaccuracies, TB measured in this way was demonstrated as the most important prognostic factor and variably correlated with most of the clinical parameters of the disease, which lost their independent predictive power whenever TB was taken into account (4). The superiority of the TB over the other single prognostic factors and composite prognostic scores was confirmed by a more accurate assessment of TB through the evaluation of the whole-body staging computed tomography (CT) $(5,6)$. This evaluation technique is direct, quantitative, and more easily reproducible than that adopted by Specht et al but it is not much simpler. It is probably for this reason that in spite of its heuristic interest and clinical advantages, with particular reference to the relationship demonstrated with the efficacy of treatments (7), this technique has not been widely applied.

Here we propose an easy, approximate estimate of TB, which can be computed quickly with a pocket calculator from some data of the patient's initial clinical evaluation, hoping 
that it could be extensively applied in patients with Hodgkin's lymphoma, whenever the direct measure seems to be unfeasible or too complicated.

\section{Patients and methods}

Patients. Over the last 12 years we retrospectively measured the relative tumor burden (rTB), the tumor burden normalized to body surface area, of 507 patients with biopsy-proven, untreated Hodgkin's lymphoma who entered some treatment protocols of the Gruppo Italiano Studio Linfomi (GISL). Fifty-one patients with early, favorable-stage disease were treated with combined VBM chemotherapy (vinblastine, bleomycin and methotrexate) and involved-field radiotherapy. One hundred and twenty-nine patients with early unfavorable-stage disease were treated with a flexible program including ABVD chemotherapy (doxorubicin, bleomycin, vinblastine and dacarbazine) and involved-field radiotherapy. After a block of three ABVD cycles these subjects underwent an early restaging, then further received either one or three ABVD cycles according to the recorded complete or partial response, respectively. Another 327 patients with advanced-stage disease entered two subsequent randomized trials in which an identical arm with ABVD six cycles plus optional radiotherapy constituted the reference standard treatment, which was administered to 117 patients. The experimental treatments included different chemotherapy regimens, invariably administered in six cycles followed by the same optional and limited radiotherapy as in the ABVD arm. The regimens were six cycles of $\mathrm{M}(\mathrm{C})$ OPPEBVCAD [mechlorethamine (or cyclophosphamide), vincristine, procarbazine, prednisone, epidoxorubicin, bleomycin, vinblastine, lomustine, melphalan and vindesine] in 103 patients and BEACOPP (bleomycin, etoposide, doxorubicin, cyclophosphamide, vincristine, procarbazine and prednisone) in 107. Details on staging, treatment and clinical results of the trials that included the patients of the present study were reported by Gobbi et al (8) for those with early favorable stages, by Iannitto et al (9) for the patients with early unfavorable stages, by Gobbi et al (10) and Federico et al (11) for patients with advanced-stage disease.

Staging procedures were performed according to the Cotswolds' Meeting (12) recommendations. The criteria for inclusion of patients into the present retrospective study were the following: availability of the magnetic records of CT scans of the neck, thorax, abdomen and pelvis performed before the start of treatment; availability of information regarding presence of bulky mass, constitutional symptoms, number of involved lymph nodes and extralymphatic involvement; availability of a complete data set regarding differential blood count [white blood cells (WBC), lymphocyte (Ly)], hemoglobin concentration $(\mathrm{Hb})$, eryhtrocyte sedimentation rate (ESR), serum albumin (Alb), serum lactate dehydrogenase (LDH), serum $\beta 2$-microglobulin ( $\beta 2-\mathrm{m}$ ); Karnofsky index; pattern of bone marrow infiltration, if present (nodular or focal or diffuse); patients' signed consent to the use of these radiologic and clinical data. The International Prognostic Index (IPI) score (13) was determined for each patient. As indicated by Vassilakopoulos et al (14), the anatomical areas of involvement that were considered for numbering were the following: Waldeyer's ring; cervical and/or supraclavicular and/or occip- ital and/or preauricular (right and left separately); axillary and/ or infraclavicular (right and left separately); epitrochlear (right and left separately); mediastinal (one site); hilar (right and left separately); paraortic (one site); coeliac and hepatic hilar nodes (one site); mesenteric (one site); iliac and/or inguinal (right and left separately); spleen and/or splenic hilar nodes (one site); popliteal (right and left separately). Each extranodal site of involvement was considered separately and added to nodal ones. Each lung was considered as a separate site. Bone marrow was also considered as one site of involvement even in the case of multiple lesions. A mediastinal mass greater than one-third of the maximum diameter of the chest at T5-T6 level and/or peripheral or retroperitoneal lymphadenopathy $>10 \mathrm{~cm}$ in the largest diameter were considered bulky masses (12).

Tumor burden assessment. The technical procedures for assessing tumor burden from CT scans have been detailed carefully elsewhere $(5,6)$. The CT imaging had to be performed before the start of the treatment, had to cover the neck, thorax, abdomen and pelvis, and images had to be taken with nonionic contrast medium. The majority of CT scans were saved on magnetic records and were re-evaluated by means of either the software resources available in the CT equipment or the Osirix ${ }^{\circledR}$ software for Apple computers. Radiologists, who were always blind to any clinical information about the patients, systematically outlined every lymphomatous lesion, nodal or extranodal, in each scan slice. This allowed calculation of the areas of the lesions which were present in any slice; from the thickness of the slices the volume of the tumor per slice and, finally, the sum of the volumes in all the slices. The volume of bone marrow involved was calculated from the volume of hemopoietically active tissue using the simple Wickramasinghe's formula (15) (hemopoietic bone marrow $=20 \mathrm{ml} / \mathrm{kg}$ body weight) applied to the ideal body weight according to Devine (16) [ideal body weight $=50 \mathrm{~kg}$ (for males) or $43.5 \mathrm{~kg}$ (for females) $+2.3 \mathrm{~kg}$ per inch $(2.54 \mathrm{~cm})$ of height $>5$ feet $(152.4 \mathrm{~cm})]$. A variable fraction of the so calculated total marrow volume was taken into consideration for the addition to the whole tumor burden according to the microscopic pattern of the lymphoid infiltration, either diffuse $(50 \%)$ focal (10\%) or nodular (5\%) (4). Finally, the obtained TB was normalized to the body surface area and this relative tumor burden (rTB), expressed in $\mathrm{cm}^{3} / \mathrm{m}^{2}$, was utilized throughout the study with two aims. First, to relate the patient's metabolic and immunologic functions and parameters to the proportion of TB with the size of the host rather than to the absolute tumor load and, second, to ensure comparability with the amount of antineoplastic drugs (usually undergoing the same normalization).

Statistics. A series of simple and multiple regression analyses with the elementary staging parameters as predictive variables and the measured rTB as the dependent variable were conducted to search for a simple combination of clinical parameters that could estimate the total tumor volume with enough accuracy. The $\mathrm{R}^{2}$, as an index of predictive ability, and the inferential tests on the partial regression coefficients, as a measure of correlation, were the guides for selecting the best variables. The dummy values ' 0 ' and ' 1 ' were assigned according to the absence or presence, respectively, of the nominal variables 
Table I. Main clinical characteristics of the whole patient population, of the training subgroup and of the test subgroup.

\begin{tabular}{|c|c|c|c|}
\hline & $\begin{array}{c}\text { Whole } \\
\text { population }\end{array}$ & $\begin{array}{l}\text { Training } \\
\text { subgroup }\end{array}$ & $\begin{array}{c}\text { Test } \\
\text { subgroup }\end{array}$ \\
\hline Number of patients & 507 & 254 & 253 \\
\hline Gender $(\mathrm{M} / \mathrm{F})$ & $253 / 254$ & $131 / 123$ & $122 / 131$ \\
\hline Age $(\text { year })^{\mathrm{a}}$ & $34.3 \pm 15.4$ & $34.4 \pm 15.2$ & $34.3 \pm 15.7$ \\
\hline Stage (I/II/III/IV) & $55 / 274 / 105 / 73$ & $28 / 130 / 56 / 40$ & $27 / 144 / 49 / 33$ \\
\hline \multicolumn{4}{|l|}{ Histological type } \\
\hline LP/LRHL/NS & $20 / 8 / 361$ & $11 / 3 / 177$ & $9 / 5 / 184$ \\
\hline MC/LD/unclassifiable & $94 / 17 / 7$ & $48 / 10 / 5$ & $46 / 7 / 2$ \\
\hline 'B' symptoms & 210 & 100 & 110 \\
\hline Bulky mass & 169 & 84 & 85 \\
\hline Bone marrow involvement & 30 & 17 & 13 \\
\hline Extranodal involvement & 161 & 90 & 71 \\
\hline \multicolumn{4}{|l|}{ No. of involved sites } \\
\hline$\leq 2 / 3-5$ & $180 / 236$ & $84 / 131$ & $96 / 105$ \\
\hline $6-10 />10$ & $86 / 5$ & $37 / 2$ & $49 / 3$ \\
\hline IPI score $(\leq 2 / \geq 3)$ & $379 / 128$ & $191 / 63$ & $188 / 65$ \\
\hline $\operatorname{ESR}(\mathrm{mm} / \text { first hour })^{\mathrm{a}}$ & $49.9 \pm 34.8$ & $49.2 \pm 34.4$ & $50.7 \pm 35.2$ \\
\hline $\mathrm{Hb}(\mathrm{g} / \mathrm{dl})^{\mathrm{a}}$ & $12.4 \pm 1.8$ & $12.4 \pm 1.8$ & $12.4 \pm 1.9$ \\
\hline $\operatorname{Albumin}(\mathrm{g} / \mathrm{dl})^{\mathrm{a}}$ & $3.89 \pm 0.60$ & $3.90 \pm 0.60$ & $3.89 \pm 0.59$ \\
\hline $\mathrm{LDH}(\mathrm{U} / \mathrm{ml})^{\mathrm{a}}$ & $382 \pm 188$ & $382 \pm 170$ & $382 \pm 200$ \\
\hline$\beta 2$-microglobulin $(\mathrm{U} / \mathrm{l})^{\mathrm{a}}$ & $2.28 \pm 1.53$ & $2.40 \pm 1.94$ & $2.17 \pm 1.12$ \\
\hline WBC count $\left(10^{9} / 1\right)^{\mathrm{a}}$ & $10.5 \pm 4.2$ & $10.3 \pm 4.4$ & $10.8 \pm 4.0$ \\
\hline Lymphocyte count $\left(10^{9} / 1\right)^{\mathrm{a}}$ & $1.7 \pm 1.2$ & $1.8 \pm 1.5$ & $1.8 \pm 1.0$ \\
\hline
\end{tabular}

LP, lymphocyte predominance; LRHL, lymphocyte-rich Hodgkin's lymphoma; NS, nodular sclerosis; MC, mixed cellularity; LD, lymphocyte depletion; IPI, international prognostic index; ESR, erythrocyte sedimentation rate; Hb, hemoglobin; LDH, lactate dehydrogenase; WBC, white blood cell. ${ }^{a}$ The results are the mean \pm SD .

(bulky mass, 'B' symptoms, extranodal involvement, bone marrow involvement, gender) (17). The final objective was to determine the set of a few clinical variables with the highest predictive value of the measured rTB.

The study population was subdivided into two independent subsets of patients. We explored first the training sample of 254 subjects, and comparatively evaluated the predictive ability of the elementary staging parameters and selected the best combination. In the second subset involving 253 patients, we cross-validated the predictive accuracy of the selection made in the first group. The patients were subdivided by assigning alternate patients to the two groups following the chronological order of recruitment into the study.

The failure-free survival was computed from the beginning of treatment up until one of the following events: disease progression during treatment, incomplete remission at the end of treatment, relapse or death from the disease.

\section{Results}

Table I illustrates the clinical characteristics of the patients studied and displays that both the training and test sample were well-balanced.
The evaluation of the simple regressions towards the measured rTB of the 20 staging parameters listed in Table II demonstrated that most of the variables were statistically correlated with the rTB at a highly significant level whereas their individual $\mathrm{R}^{2}$ is generally low, apart from that of the IPI score, due to its multiparametric characteristic. The data presented in Table II indicated that the possibility of improving the prediction of rTB made with a single parameter by combining a certain number of parameters would be worth exploring.

A series of multiple regressions selected only the following three staging variables strictly correlated with rTB: IPI score, bulky mass and number of involved sites (a multiple regression with these three variables had an $\mathrm{R}^{2}$ of 0.496 ). A careful evaluation of the relationship between these three variables and rTB (Table III) showed that the best regression of the IPI score with rTB is not linear, but quadratic $\left(\mathrm{R}^{2}=0.464\right.$ vs. 0.378$)$. Moreover, the compared analysis of the regression coefficients showed that the presence of a bulky mass corresponds, on average and as far as the prediction of rTB is concerned, to about three additional involved sites besides those actually recorded.

The multiple regression with these two transformed variables, the squared IPI and the number of involved sites augmented by three in case of bulky mass, reached an $\mathrm{R}^{2}$ of 
Table II. Simple regressions of each clinical variable vs. the relative tumor burden (rTB) in the training subgroup of 254 patients.

\begin{tabular}{|c|c|c|c|}
\hline & Coefficient & P-value & $\mathrm{R}^{2}$ \\
\hline IPI score & 58.167 & $<0.0001$ & 0.378 \\
\hline Bulky mass & 113.507 & $<0.0001$ & 0.186 \\
\hline Extranodal involvement & 81.875 & $<0.0001$ & 0.113 \\
\hline $\mathrm{Hb}(\mathrm{g} / \mathrm{dl})$ & -22.168 & $<0.0001$ & 0.107 \\
\hline $\operatorname{ESR}(\mathrm{mm} / 1 \mathrm{~h})$ & 1.116 & $<0.0001$ & 0.097 \\
\hline No. of involved sites & 16.361 & $<0.0001$ & 0.089 \\
\hline 'B' symptoms & -74.431 & $<0.0001$ & 0.086 \\
\hline Stage & 41.592 & $<0.0001$ & 0.082 \\
\hline Karnofsky index & -3.101 & $<0.0001$ & 0.076 \\
\hline Serum LDH (U/1) & 0.175 & $<0.0001$ & 0.069 \\
\hline Serum albumin $(\mathrm{g} / \mathrm{dl})$ & -49.699 & $<0.0001$ & 0.059 \\
\hline $\operatorname{WBC}\left(10^{9} / 1\right)$ & 0.005 & 0.0002 & 0.026 \\
\hline Serum $\beta 2$-microglobulin $(\mu \mathrm{g} / \mathrm{l})$ & 0.013 & 0.0102 & 0.025 \\
\hline Bone marrow involvement & 80.314 & 0.0006 & 0.023 \\
\hline Age (years) & -1.138 & 0.0015 & 0.020 \\
\hline Gender $(\mathrm{M} / \mathrm{F})$ & 25.663 & 0.0207 & 0.011 \\
\hline Lymphocytes $\left(10^{9} / 1\right)$ & -0.010 & 0.0254 & 0.010 \\
\hline Histological type & 11.311 & 0.0543 & 0.007 \\
\hline Lymphocytes (\%) & -0.001 & 0.9508 & 0.000 \\
\hline
\end{tabular}

IPI, international prognostic index; Hb, hemoglobin; ESR, erythrocyte sedimentation rate; LDH, lactate dehydrogenase; WBC, white blood cell.

Table III. Bivariate regressions with rTB of the IPI and the IPI $^{2}$ score, then of the bulky mass and of the number of involved sites.

\begin{tabular}{|c|c|c|c|c|}
\hline & Coefficient & SE & T-value & P-value \\
\hline \multicolumn{5}{|c|}{ Bivariate regression with rTB of IPI and IPI ${ }^{2}$} \\
\hline Intercept & 66.524 & 9.964 & 6.676 & $<0.0001$ \\
\hline IPI & 18.129 & 8.868 & 2.044 & 0.0414 \\
\hline $\mathrm{IPI}^{2}$ & 8.508 & 1.753 & 4.853 & $<0.0001$ \\
\hline \multicolumn{5}{|c|}{ Bivariate regression with $\mathrm{rTB}$ of bulky mass and the no. of involved sites } \\
\hline Intercept & 6.882 & 7.141 & 0.964 & 0.3356 \\
\hline Bulky mass & 94.558 & 7.537 & 12.547 & $<0.0001$ \\
\hline No. involved sites & 28.147 & 1.584 & 17.764 & $<0.0001$ \\
\hline
\end{tabular}

IPI, international prognostic index.

0.673 in the training sample and 0.661 in the test sample. The following equation, $\left[-4.3+8.3 \times \mathrm{IPI}^{2}+22.7 \times\right.$ no. of involved sites ( +3 if bulky mass is present)], drawn from the multiple regressions, was used to calculate an estimated rTB (Es-rTB) in all cases. This, in turn, showed a highly significant correlation with the measured rTB, with an $\mathrm{R}^{2}$ of 0.878 in the training sample (95\% confidence interval: $0.827-0.919)$ and 0.844 in the test sample (95\% confidence interval: 0.790-0.898). The accuracy of the Es-rTB derived in this way seemed to be acceptable, since the error of the estimate is lower than $50 \%$ of the mean value of $\mathrm{rTB}\left(143.4 \mathrm{~cm}^{3} / \mathrm{m}^{2}\right)$ in $81 \%$ of the cases of the training sample and in $77 \%$ of those of the test sample.
Most convincingly, the Es-rTB retained a better prognostic discrimination than the IPI score alone in terms of failure-free survival both in the training sample and in the test sample (Fig. 1). This result shows that the Es-rTB can be an easy prognostic tool for Hodgkin's lymphoma, one that is much simpler than the measured rTB, and sufficiently accurate and reliable for clinical purposes.

\section{Discussion}

If the total tumor burden is considered as the whole amount of the truly malignant cells (the Reed-Sternberg and the 
TRAINING SAMPLE
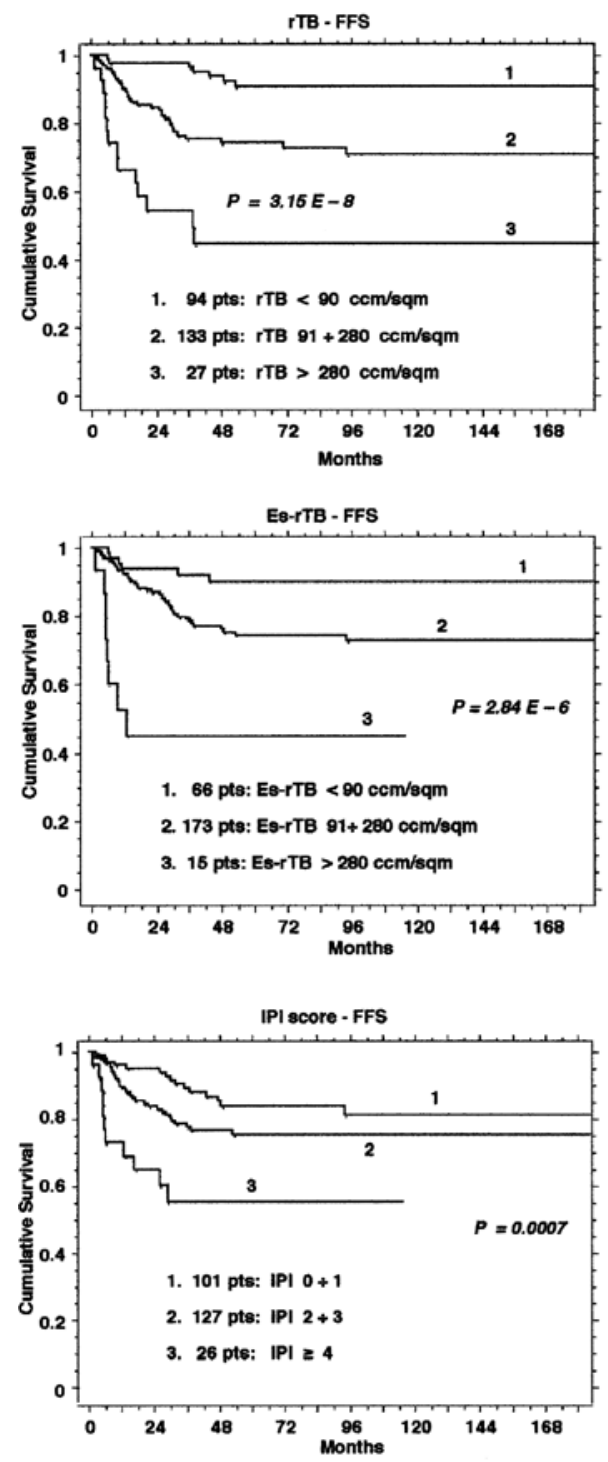

TEST SAMPLE
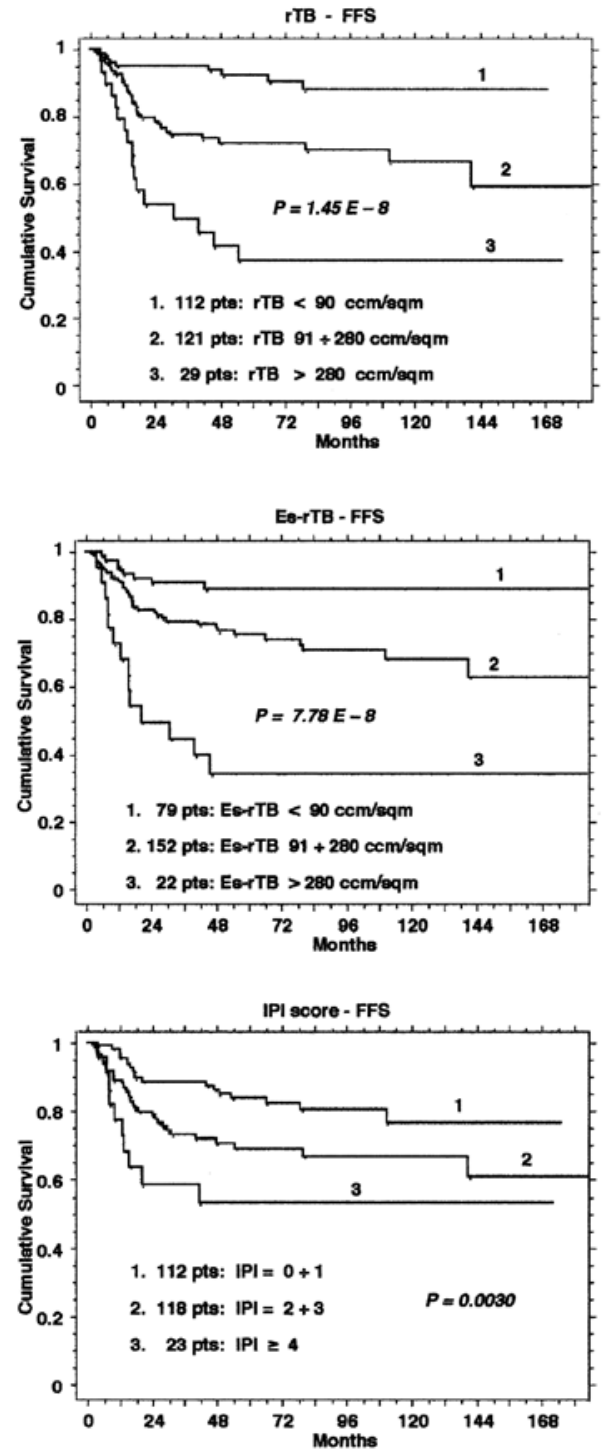

Figure 1. Comparison of the predictive power, relative to the failure-free survival (FFS), of the measured rTB, the estimated rTB (Es-rTB) and the IPI score both in the training subgroup (254 patients) and in the test subgroup (253 patients).

Hodgkin cells) this should correspond to a small component of the pathologic tissue of Hodgkin's lymphoma. This is mostly represented by a variable mixture of reactive, non-neoplastic small lymphocytes, eosinophils, neutrophils, histiocytes and plasma cells, and precisely for this reason it would appear that the quantification of the purely neoplastic component might be more interesting and useful than evaluations including the reactive component.

However, although it seems unquestionable that the neoplastic cells are the primary determinants of the biological and clinical alterations, both local and systemic, ultimately conditioning the prognosis, quantification of the purely neoplastic cells, when performed, did not clearly improve predictive ability (2) over computation of the whole cell population. This might mean that the variable intensity of the biological activity of the tumor cells is more important than their absolute number, and that their cytokine production and consequent immunologic interactions are crucial for the recruitment of a variably abundant mass of reactive non-neoplastic cells. These constitute a larger proportion of the volume of the clinically evident lesions compared to the neoplastic elements by which they are directly or indirectly stimulated, and the biological activity of which they reflect. Thus, it seems reasonable that, for clinical and prognostic purposes, it may be less important to assess the neoplastic component separately from the reactive one. In fact, in clinical practice therapeutic decisions are based on the whole size of the lesions, as evaluated by palpation, ultrasonography, CT and positron emission tomography. These techniques do not discriminate between neoplastic and reactive cell populations.

Some comments are needed to clarify the aspects of the equation used to estimate the rTB. It is essentially based on two parameters, the number of involved sites, which can also incorporate information on the presence of bulky mass, and the IPI score. The number of involved sites was pointed out as a partial surrogate parameter of tumor burden by 
Vassilakopoulos et al (14) and its importance was partially confirmed by our results. Specifically, in the prognostic comparison with the measured rTB performed in a previous analysis (4), the number of involved sites was found to be the second best factor related to the time-to-treatment failure and to disease-free survival and the third best factor related to less than complete response or relapse within 12 months of the end of the treatment. The definition of the anatomic regions for the number of involved sites as suggested by Vassilakopoulos et al (14) has been demonstrated to be prognostically valid. In this study the average prognostic correspondence of bulky mass to about three further involved sites besides those actually recorded is an indirect confirmation of its value.

The paramount predictive power of the IPI score related to the rTB is likely due to the score's integration of seven elementary prognostic parameter (serum albumin $<4 \mathrm{~g} / \mathrm{dl}$, hemoglobin $<10.5 \mathrm{~g} / \mathrm{dl}$, male gender, stage IV, age $\geq 45 \mathrm{yr}$, white cell count $\geq 15,000 / \mu 1$ and lymphocyte count $<600 / \mu 1$ or $<8 \%$ ), nearly all of which are independently related to the rTB (see Table II). The inclusion of the IPI score in the equation was fully expected. However, its quadratic regression with the measured rTB was unexpected and indicates that the presence of any additional parameter within the IPI score is supported by an additional, rather than constant, increase of the amount of tumor. This provides further evidence of the relationship between the activity of the neoplastic component and the effects of its cytokine production, both locally, with recruitment of reactive cells, and systemically, with functional depression of liver and bone marrow (as reflected by many of the IPI variables).

Despite the elevated $\mathrm{R}^{2}$ the equation has a notable estimate error, which cannot be further reduced by other available pre-therapeutic parameters. Nevertheless, the estimated rTB retained a significant prognostic advantage over the IPI score. Moreover, as recently pointed out, the estimated rTB could help to identify the possibility of response to a given treatment in relation to the initial rTB (5). Thus, the indirect estimate of rTB can be proposed for investigational and clinical use when a direct measurement cannot be performed, at least until a new, simplified technique for the direct assessment of rTB becomes available.

\section{Acknowledgements}

This study was supported in part by grants from the Fondazione IRCCS Policlinico S. Matteo, Pavia, and from the 'Ferrata-Storti Foundation', Pavia. We are indebted to Dr Rachel Stenner for her careful assistance with English language editing of this manuscript.

\section{References}

1. Specht L: Prognostic factors in Hodgkin's disease. Dan Med Bull 39: 409-422, 1992.
2. Josting A: Prognostic factors in Hodgkin lymphoma. Expert Rev Hematol 3: 583-592, 2010.

3. Specht L and Nissen NI: Prognostic significance of tumor burden in Hodgkin's disease PS I and II. Scand J Haematol 36: 367-375, 1986.

4. Specht L: Tumor burden as the main indicator of prognosis in Hodgkin's disease. Eur J Cancer 28: 1982-1985, 1992.

5. Gobbi PG, Ghirardelli ML, Solcia M, Di Giulio G, Merli F, Tavecchia L, Bertè R, Davini O, Levis A, Broglia C, et al: Imageaided estimate of tumor burden in Hodgkin's disease: evidence of its primary prognostic importance. J Clin Oncol 19: 1388-1394, 2001.

6. Gobbi PG, Broglia C, Di Giulio G, Mantelli M, Anselmo P, Merli F, Zinzani PL, Rossi G, Callea V, Iannitto E, et al: The clinical value of tumor burden at diagnosis in Hodgkin lymphoma. Cancer 101: 1824-1834, 2004.

7. Gobbi PG, Valentino F, Bassi E, Coriani C, Merli F, Bonfante V, Marchianò A, Gallamini A, Bolis S, Stelitano C, et al: Chemoresistance as a function of the pretherapy tumor burden and the chemotherapy regimen administered: differences observed with two current chemotherapy regimens for advanced Hodgkin lymphoma. Clin Lymphoma Myeloma Leuk 5: 396-402, 2011.

8. Gobbi PG, Broglia C, Merli F, Dell'Olio M, Stelitano C, Iannitto E, Federico M, Bertè R, Luisi D, Molica S, Cavalli C, Dezza L and Ascari E: Vinblastine, bleomycin and methotrexate chemotherapy plus irradiation for patients with early-stage, favorable Hodgkin's lymphoma: the experience of the Gruppo Italiano Studio Linfomi (GISL). Cancer 98: 2393-2401, 2003.

9. Iannitto E, Minardi V, Gobbi PG, Calvaruso G, Tripodo C, Marcheselli L, Luminari S, Merli F, Baldini L, Stelitano C, et al: Response-guided ABVD chemotherapy plus involved-field radiation therapy for intermediate-stage Hodgkin lymphoma in the pre-positron emission tomography era: a Gruppo Italiano Studio Linfomi (GISL) prospective trial. Clin Lymphoma Myeloma 9: 138-144, 2009.

10. Gobbi PG, Levis A, Chisesi T, Broglia C, Vitolo U, Stelitano C, Pavone V, Cavanna L, Santini G, Merli F, et al: ABVD versus modified Stanford V versus MOPPEBVCAD with optional and limited radiotherapy in intermediate- and advanced-stage Hodgkin's lymphoma: final results of a multicenter randomized trial by the Intergruppo Italiano Linfomi. J Clin Oncol 23: 9198-9207, 2005.

11. Federico M, Luminari S, Iannitto E, Polimeno G, Marcheselli L, Montanini A, La Sala A, Merli F, Stelitano C, Pozzi S, et al: ABVD compared with BEACOPP compared with CEC for the initial treatment of patients with advanced Hodgkin's lymphoma: results from the HD2000 Gruppo Italiano per lo Studio dei Linfomi Trial. J. Clin Oncol 27: 805-811, 2009.

12. Lister TA, Crowther D, Sutcliffe SB, Glatstein E, Canellos GP, Young RC, Rosenberg SA, Coltman CA and Tubiana M: Report of a committee convened to discuss the evaluation and staging of patients with Hodgkin's disease: Cotswolds meeting. J Clin Oncol 7: 1630-1636, 1989.

13. Hasenclever D and Diehl V: A prognostic score for advanced Hodgkin's disease. International Prognostic Factors Project on Advanced Hodgkin's Disease. N Engl J Med 339: 1506-1514, 1998.

14. Vassilakopoulos TP, Angelopoulou MK, Siakantaris MP, Kontopidou FN, Dimopoulou MN, Barbounis A, Grigorakis V, Karkantaris C, Anargyrou K, Chatziioannou M, et al: Prognostic factors in advanced stage Hodgkin's lymphoma: the significance of the number of involved anatomic sites. Eur J Haematol 67: 279-288, 2001.

15. Wickramasinghe SN: Human Bone Marrow. Blackwell Scientific Publications, Oxford, pp211-216, 1976.

16. Devine BJ: Gentamicin therapy. Drug Intell Clin Pharm 8: 650-655, 1974.

17. Armitage $P$ and Berry G (eds): Statistical Methods in Medical Research. 2nd edition. Blackwell Scientific Publication, Oxford, pp369-396, 1987. 